\title{
Multi-Channel Active Noise Control System Designs with Fuzzy Logic Stabilized Algorithms
}

\author{
DongJun Ahn ${ }^{1, a}$, KeunSik Kim ${ }^{1, b}$, Hyun Do Nam ${ }^{2, c}$ EunWoo Shin ${ }^{3, d}$ \\ ${ }^{1}$ Department of Automotive Engineering, Ajou Motor College, San 6-7, Kwansanri, Jupo Myeon, BoRyeong \\ $\mathrm{Si}$, ChungNam, 355-769, Korea \\ ${ }^{2}$ Department of Electronics and Electrical Engineering, Dankook University, 126, Jukjeon-dong, \\ Suji-gu, Gyeonggi-do, 448-701, Korea \\ ${ }^{3}$ Active Noise Control System R/D Center, SQ Engineering Co., Ltd., 160-11, Garak-dong, Songpa- \\ gu, Seoul, 138-160, Korea \\ aahndj@motor.ac.kr, 'kskim@motor.ac.kr, chdnam@dankook.ac.kr , 'ewshin11@hanmail.net
}

Keywords: Active Noise Control, Adaptive Filter, Stabilized Algorithm, multi channel LMS algorithm, IIR Filter, Fuzzy logic

\begin{abstract}
In active noise control filter, IIR filter structure which used for control filter assures the stability property. The stability characteristics of IIR filter structure is mainly determined by pole location of control filter within unit disc, so stable selection of the value of control filter coefficient is very important. In this paper, we proposed novel adaptive stabilized Filtered_U LMS algorithms with IIR filter structure which has better convergence speed and less computational burden than conventional FIR structures, for multi-channel active noise control with vehicle enclosure signal case. For better convergence speed in adaptive algorithms, fuzzy LMS algorithms where convergence coefficient computed by a fuzzy PI type controller was proposed.
\end{abstract}

\section{Multi-channel Active Noise control systems}

Unwanted acoustic noise problems become more and more evident as increased numbers of large industrial equipment such as engines, blowers, fans, transformers, and compressors are in use. Mechanical vibration is another related type of noise that commonly creates problems in all areas of transportation and manufacturing, as well as many household appliances.

The traditional approach to acoustic noise control use passive techniques such as enclosures, barriers, and silencers to attenuate the undesired noise. These passive silencers are valued for their high attenuation over broad frequency range, but they are relatively large, costly, and ineffective at low frequencies. The attenuation of passive silencers is low when the acoustic wavelength is large compared to the silencer dimensions, such as body length or lining thickness.

ANC systems utilizing a microphone and an electronically driven loud speaker to generate a canceling sound was first proposed in a 1936 patent by Lueg[1]. Recently, ANC systems needs highly precise control, temporal stability, and reliability performances. It is therefore desirable for noise canceller to be digital, where signals from electroacoustic or electromechanical transducers are sampled and processed using DSP systems with enough speed and precision to execute sophisticated mathematical functions in real time[2 3].

In active noise control filter, IIR filter structure which used for control filter assures the stability property. The stability characteristics of IIR filter structure is mainly determined by pole location of control filter within unit disc, so stable selection of value of control filter coefficient is very important.

In this paper, we proposed novel adaptive stabilized fuzzy Filtered U LMS algorithms with IIR filter structure which has better convergence speed and less computational burden than conventional FIR structures, for multi-channel active noise control with vehicle enclosure signal case.

Fig 1. Shows that multi-channel ANC systems using J reference sensors, K parallel adaptive filters and corresponding secondary sources, and $M$ error sensors[4]. Fig1. Can be simplified to block diagram shown in fig. 2. The elements of the vector $d(n)$ are the primary noise components of the error sensor outputs. The matrix $\mathrm{P}(\mathrm{z})$ represents $\mathrm{Mx} \mathrm{J}$ primary path transfer function, the matrix $\mathrm{S}(\mathrm{z})$ represents $\mathrm{M}$ x K secondary path transfer functions. 

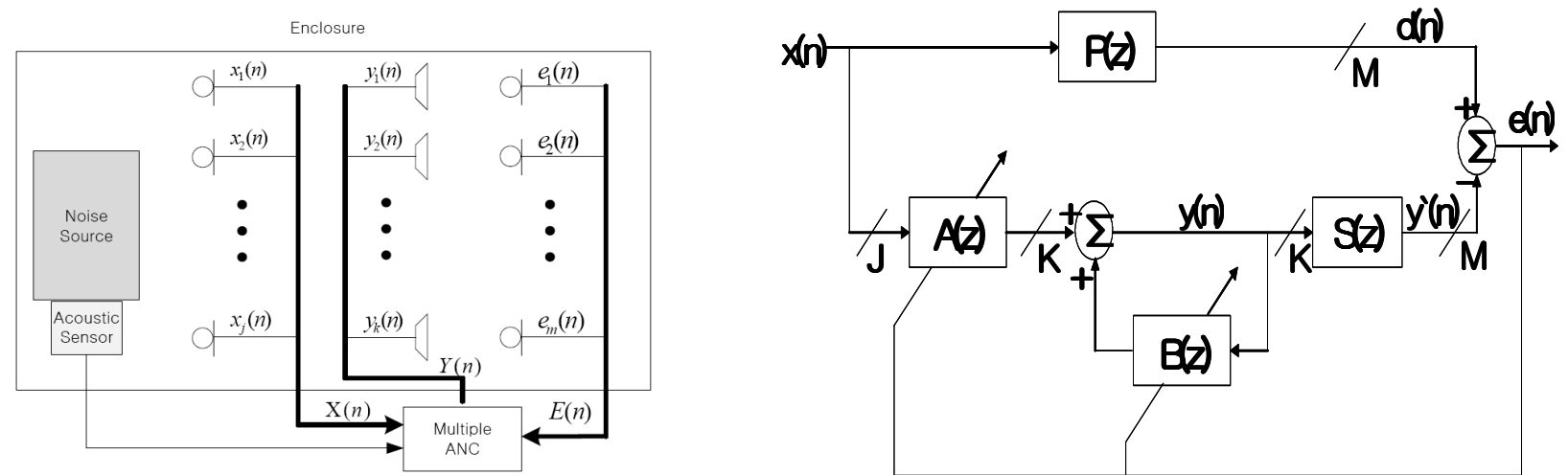

Fig. 1. 3-Dimensional Active Noise Cancelling System Fig. 2. Block diagram of multi-channel ANC System

The output of k-th secondary transfer function as follows.

$$
y_{k}(n)=\sum_{j=1}^{J} a_{k j}^{T}(n) x_{j}(n)+\sum_{i=1}^{K} b_{k i}^{T}(n) y_{i}(n-1)
$$

for $k=1, \quad 2, \cdots, K$, where

$$
\begin{aligned}
& a_{k j}(z) \equiv a_{k j, 0}(n)+a_{k j, 1}(n) z^{-1}+a_{k j, 2} z^{-2}+\cdots+a_{k j, L-1}(n) z^{L-1} \\
& b_{k i}(z)=b_{k i, 1}(n) z^{-1}+b_{k i, 2}(n) z^{-2}+b_{k i, 3}(n) z^{-3}+\cdots+b_{k i, I}(n) z^{I}
\end{aligned}
$$

For unknown or time varying equations of adaptive LMS algorithms is as follows.

$$
\begin{aligned}
& a_{k j}(n+1)=a_{k j}(n)+\mu_{a} \sum_{m=1}^{M} x_{j k m}^{\prime}(n) e_{m}(n) \\
& b_{k i}(n+1)=b_{k i}(n)+\mu_{b} \sum_{m=1}^{M} y_{i k m}^{\prime}(n) e_{m}(n)
\end{aligned}
$$

where

$$
\begin{aligned}
& x_{j k m}^{\prime}(n) \equiv \hat{S}_{m k}(n) * x_{j}(n) \\
& y_{i k m}^{\prime}(n) \equiv \hat{S}_{m k}(n) * y_{i}(n)
\end{aligned}
$$

are respectively, the filtered reference and output signal vectors. In order to get optimal control filter coefficients, must be consider the effect of secondary path transfer functions $\hat{S}_{m k}(n)$, and then Fig. 2 modified as Fig. 3( $\mathrm{J}=1, \mathrm{M}=2, \mathrm{~K}=2)$. These methods are called by Filtered_U LMS algorithms.

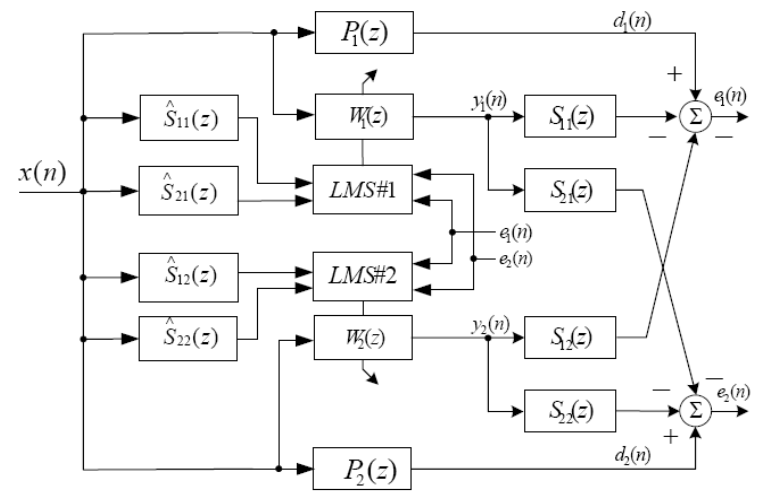

Fig. 3. Multi-channel $(1 \times 2 \times 2)$ ANC System

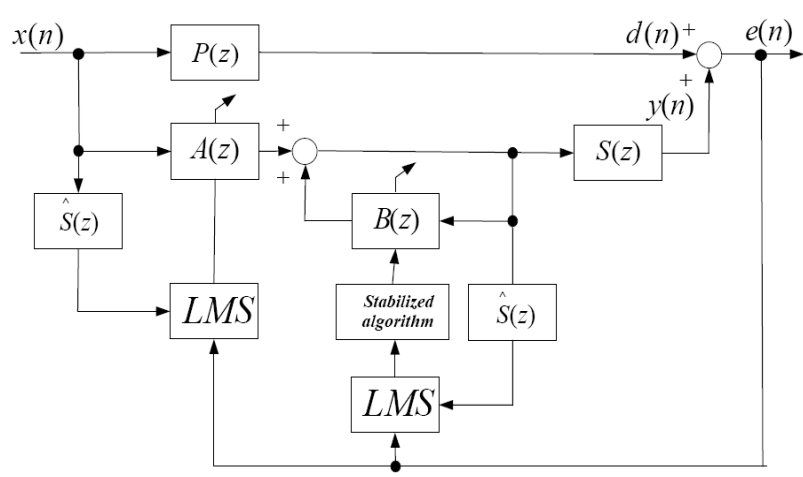

Fig. 4. Stabilized Filtered_U ANC System 


\section{Stabilized IIR filter structure}

An adaptive IIR filter structure was considered the feedback as a part of the overall plant, it allows the system to dynamically track changes in the secondary and feedback paths during cancellation operations. But Eq. 6 implies the adaptive IIR filter would be try to generate poles corresponding to the ideal response, and since the trajectory of the filter poles during convergence is rather unpredictable, one or several poles could be more outside the unit circle, leading to instability. The problem with unstable filter poles can be solved such as leaky LMS algorithm[5]. However, This methods has slow convergence characteristics of adaptive algorithms.

In this paper, we proposed the stabilized algorithms for adaptive IIR filter structure[6 7] with multi-channel ANC systems which shows in Fig. 4. Eq. 7 represent proposed algorithms that pre-filter polynomial matrix $\mathrm{F}(\mathrm{z})$ which multiply by control filter polynomial matrix $\mathrm{B}(\mathrm{z})$. Pre-filter polynomial $\mathrm{f}(\mathrm{z})$ has a role that moving the unstable filter poles in transient state, and then return originally in steady state.

$$
\mathbf{F}(z)=\left[\begin{array}{cccc}
f_{11}(z) & f_{12}(z) & \cdots & f_{1 K}(z) \\
f_{21}(z) & \cdots & \cdots & f_{2 K}(z) \\
\vdots & \ddots & \ddots & \vdots \\
f_{K 1}(z) & \cdots & \cdots & f_{K K}(z)
\end{array}\right]=\mathbf{I}_{K}-k \mathbf{B}(z)
$$

where $\mathbf{I}_{k}$ is $\mathrm{K} x \mathrm{~K}$ unit matrix, and then pre-filter polynomial is

$$
f_{k k}(z)=1+k(n) b_{k k, 1}(n) z^{-1}+k^{2}(n) b_{k k, 2}(n) z^{-2}+\cdots+k^{I}(n) b_{k k, I}(n) z^{-I}
$$

where $0 \leq \boldsymbol{k} \leq 1$, calculation of coefficient $\mathrm{k}$ is as follows

$$
k(n+1)=\lambda k(n)+(1-\lambda) \quad, \quad k(0)=0, \quad 0 \leq \lambda \leq 1
$$

\section{Fuzzy logic LMS algorithm}

LMS algorithms which were used for control filters, assure the convergence property, and computational burden of these algorithms are proportionate to the number of filter taps. However, slow convergence speed of the LMS algorithm is a major disadvantage. The convergence speed of the LMS algorithm is mainly determined by the value of the convergence coefficient, so optimal selection of the value of the convergence coefficient is very important. Convergence speed of the normalized LMS algorithm is relatively faster than the conventional LMS algorithm[8].

In this paper, a fuzzy LMS algorithm where the convergence coefficient is computed by a fuzzy proportional controller was proposed[9] in Fig. 5.

The estimation errors and mis-adaptation degrees were used for input of the fuzzy logic LMS algorithm in Eq. (11). Proposed fuzzy logic LMS algorithms is

$$
W(n+1)=W(n)+\frac{v(n)}{(L+1)} e(n) x(n)
$$

where $v(n)$ is output of fuzzy inference engine, and $\mathrm{L}$ is order of control filter.

Calculation procedures of $v(n)$ is as follows.

1) Calculate the estimation error and change of error as follows in Eq. 12 13.

$$
\begin{aligned}
& u e(n)=\alpha_{1} u e(n-1)+\left(1-\alpha_{1}\right) \frac{\|e(n) x(n)\|}{(L+1)} \\
& \left.\operatorname{uce}(n)=\alpha_{1} u c e(n-1)+\left(1-\alpha_{1}\right) \| u e(n)\right)-u e(n-1) \|
\end{aligned}
$$


where $\alpha_{1}$ is forgetting factor of $u e(n), u e(n)$ is quantitative measurement of misadaptation which obtained by norm of cross correlations of input signal and estimation error signal vector, and uce $(n)$ is result of smoothing filter which norm of change of error signal ue(n).

2) Define the membership function of fuzzy input/output variable ue $(n), u c e(n), v(n)$, respectively, as shown Fig. 6.

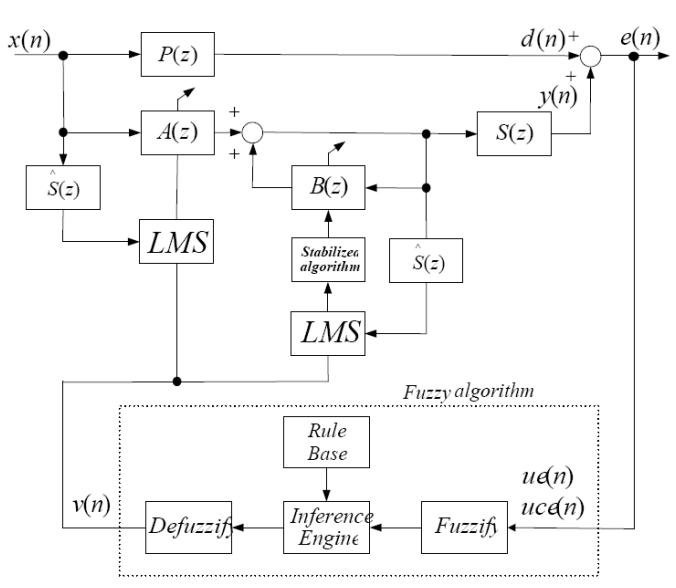

Fig. 5 Stabilized adaptive fuzzy LMS ANC algorithm

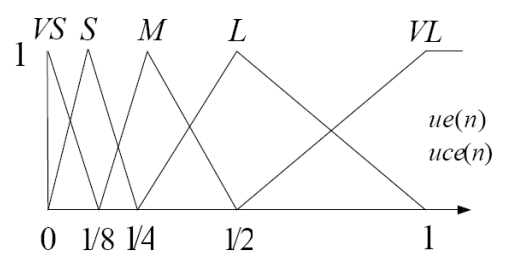

(a) $m_{u e}(U E), m_{u c e}(U C E)$

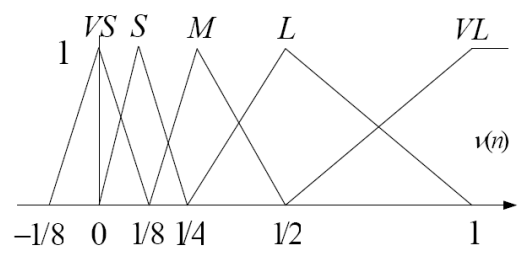

(b) $m_{v}(v)$

Fig. 6 Membership function of fuzzy input/output variables

3) Define the linguistic control rule for decision of convergence coefficient that fast convergence characteristics as Table 1.

Table 1 Linguistic control rules for decision of convergence coefficient

\begin{tabular}{|c|c|c|c|c|c|}
\hline uce ue & VS & S & M & L & XL \\
\hline VS & VL & L & M & S & VS \\
\hline S & VL & VL & M & M & S \\
\hline M & VL & VL & L & L & M \\
\hline L & VL & VL & VL & VL & L \\
\hline XL & VL & VL & VL & VL & VL \\
\hline
\end{tabular}

where VS : very small, $\mathrm{S}$ : small, $\mathrm{M}$ : medium L : large, XL : very large, respectively.

4) Calculate max-min composition operation

$$
v^{0}(n)=\max \left[\sum_{i=1}^{N} \min \left[m_{U E i}\left(u e_{i}\right), m_{U C E i}\left(u e_{i}\right), m_{v i}\left(v_{i}\right)\right]\right]
$$

where $v^{0}(n)$ is fuzzy output variable.

5) Calculate the crisp output $v(n)$, using discrete center of gravity methods (defuzzify methods)

$$
v(n)=\frac{\sum_{i=1}^{N}\left(v^{0}(n) m_{v}\left(v_{i}\right)\right)}{\sum_{i=1}^{N}\left(m_{v}\left(v_{i}\right)\right)}
$$




\section{Computer simulation}

To show effectiveness of proposed systems, computer simulations was performed by under such condition as Table 2 in given common parameters. Simulation model is applied by $1 \times 2 \times 2$ multi-channel models[4].

Table 2. Common simulation parameters

\begin{tabular}{|c|c|c|}
\hline Item & \multicolumn{2}{|c|}{ Data } \\
\hline Transfer Function & \multicolumn{2}{|c|}{$1 \times 2 \times 2$ multi model(order : 25 ) } \\
\hline Sound speed & \multicolumn{2}{|c|}{$340[\mathrm{~m} / \mathrm{s}]$} \\
\hline Sampling frequency & \multicolumn{2}{|c|}{$2[\mathrm{kHz}]$} \\
\hline Calculation step & \multicolumn{2}{|c|}{$10,000 \mathrm{step}(5 \mathrm{sec})}$. \\
\hline Reference signal & \multicolumn{2}{|c|}{ Gasoline Engine muffler } \\
\hline Estimated secondary transfer function & \multicolumn{2}{|c|}{ FIR structure, 192tap } \\
\hline Control filter tap(conventional) & \multicolumn{2}{|c|}{ FIR structure, 192tap } \\
\hline Control filter tap(proposed) & Numerator: 96 & Denumerator: 6 \\
\hline Convergence coefficient & \multicolumn{2}{|c|}{0.000015} \\
\hline
\end{tabular}

To reduce the computation burden in fuzzy inference engine that overcome real time implementation problem, we make the look-up table which composed of convergence coefficient that calculation result of fuzzy inference engine.

Fig. 7 is represented by pole-zero location plotting of transfer function of primary source and secondary sources path(system order : 25).

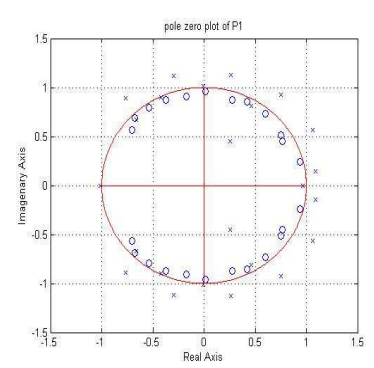

(a) primary source $\left(\mathrm{P}_{1}\right)$

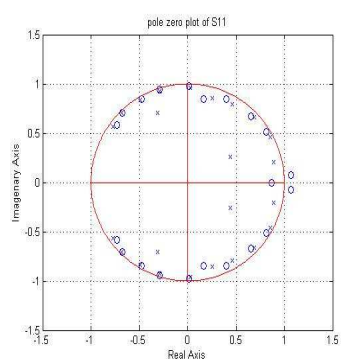

(b) secondary source $\left(\mathrm{S}_{11}\right)$

Fig. 7 Pole-zero location plotting of transfer function

Fig. 9 is simulation result of conventional algorithm and proposed algorithms, respectively. Proposed algorithm is shown that better convergence speed and computational burden than conventional algorithm.

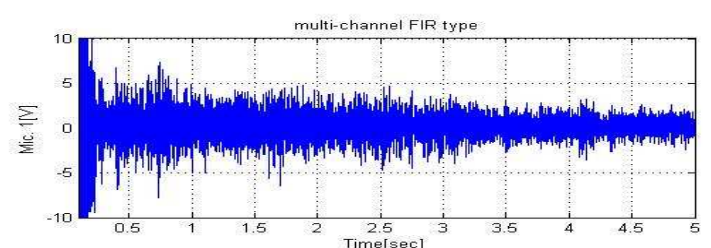

multi-channel FIR type

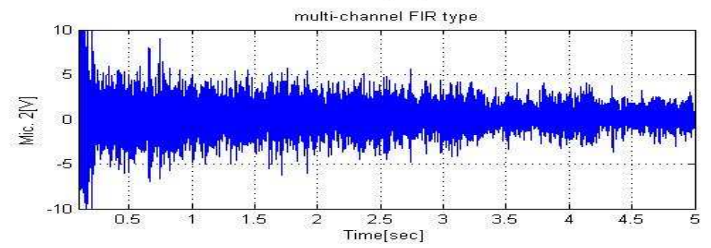

(a) conventional algorithms(FIR structure)
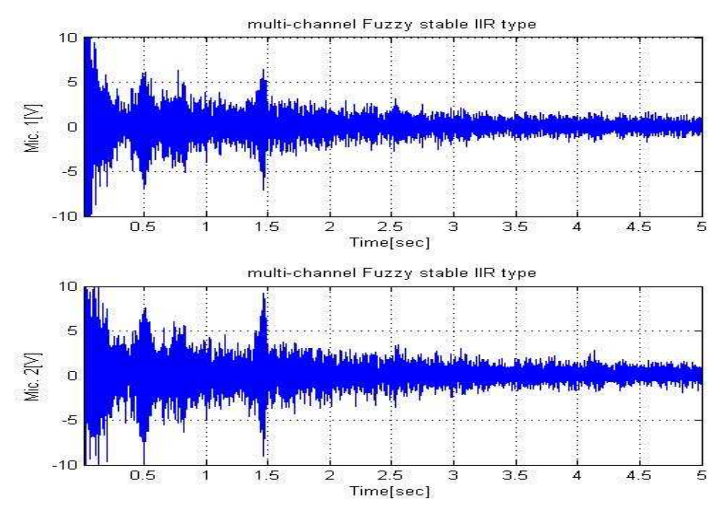

(b) proposed algorithms(Fuzzy Stable IIR)

Fig. 9 Comparison of conventional and proposed algorithms 


\section{Conclusion}

In active noise control filter, IIR filter structure which used for control filter assures the stability property. The stability characteristics of IIR filter structure is mainly determined by pole location of control filter within unit disc, so stable selection of the value of control filter coefficient is very important. In this paper, we proposed novel adaptive stabilized Filtered_U LMS algorithms with IIR filter structure which has better convergence speed and less computational burden than conventional FIR structures, for multi-channel active noise control with vehicle enclosure signal case. For better convergence speed in adaptive algorithms, fuzzy LMS algorithms was proposed where convergence coefficient computed by a fuzzy PI type controller was proposed. Computer simulations were performed to show the effectiveness of proposed algorithms with 3-dimensional model problem.

\section{Acknowledgement}

This research was financially supported by the Construction Technology Innovation Program (09 Technology Innovation E05) funded by Korea Institute of Construction \& Transportation Technology Evaluation and Planning(KICTEP) of Ministry of Land, Transportation and Maritime Affairs, Republic of Korea.

\section{References}

[1] Lueq P., "Process of silencing sound oscillations," U.S. Patent 2043, 416, (1934)

[2] Elliott S.J. and Nelson P.A., "The active control of sound," Electronics and Communication Eng. Jour., (1990) pp.127-136

[3] Nelson P.A. and Elliott S.J., "Active noise control: A tutorial review," Proceedings of the International Symposium on Active Control of Sound and Vibration, Tokyo, Japan, (1991) pp.45-74.

[4] Kuo S.M and Morgan D.R.. Active Noise Control Systems, John Wiley, 1995.

[5] Widrow B. and Stearns S.D., Adaptive Signal Processing, Englewood Cliffs, NJ: Prentice-Hall, 1985.

[6] Muhammad T. and Masahide A., Masauki K., "A New Variable Step Size LMS Algorithm-Based Method for Improved Online Secondary Path Modeling in Active Noise Control Systems," IEEE Trans. on Audio, Speech and Language Processing, (2006) Vol. 14, No. 2, 720-726.

[7] Nam H. D. and Seo S. D., Yoon K. J., Ahn D. J., "Stabilized multi-channel IIR filters for active control of noise in a duct," ICSV13-Vienna, ( 2006) Austria.

[8] Cheralu M. and Teodorescu H. N., Dumitrascu C., "A fuzzy LMS algorithm," Proceeding of the international conference on Fuzzy Logic \& Neural Networks, Japan, Isuka, (1990) pp. 107-110 .

[9] D. J. Ahn, "Design of adaptive filters for active noise control," Ph. D. theses, S. Korea, (1995) Graduate School of Dankook Univ. 